\section{Diseño y Construcción de Pistón HIDRAÚLICO}

(1) Eulogio Santos De la Cruz

(2) Oswaldo Rojas Lazo

(3) Julio Yenque Dedios

(4) Aurelio Lavado Soto

\section{RESUMEN \\ El proyecto de un sistema hidráulico, comprende el diseño, selección de materiales y construcción del pistón hidráulico, circuito hidráulico y el ensamble con la bomba y accesorios, este equipo será accionado por la fuerza de un fluido en movimiento, cuya aplicación es en los dispositivos de máquinas herramientas, estampado, perforación, empaques y otras. \\ Palabras Clave: Pistón. Hidráulica. Presión. \\ Design and Construction of a Hydraulic Piston ABSTRACT}

A hydraulic system project includes the design, materials selection and construction of the hydraulic piston, hydraulic circuit and the joint with the pump and its accesories. This equiment will be driven by the force of moving fluid, whose application is in the devices of machines, tools, printing, perforation, packing and others.

Key words: Piston. Hydraulic. Pressure.

(1) Ingeniero Industrial. Profesor del Departamento de Diseño y Tecnología Industrial, UNMSM.
E-mail: esantosd@unmsm.edu.pe

(2) Ingeniero Industrial. Profesor del Departamento de Diseño y Tecnologia Industrial, UNMSM. y Tecnologia Industrial, UNMSM.
E-mail: orojasl@unmsm.edu.pe

(3) Ingeniero Industrial. Profesor del Departamento de Diseño y Tecnologia Industrial, UNMSM. E-mail: jyenqued@unmsm.edu.pe

(4) Ingeniero Químico. Profesor del Departamento de Diseño Tecnología Industrial, UNMSM. E-mail: mlavados@unmsm.edu.pe
I NTRODUCCIÓN

El proyecto diseño de un sistema hidráulico, comprende el diseño y construcción de un pistón hidráulico, circuito hidráulico y el ensamble con la bomba y sus accesorios, que en conjunto constituyen un sistema generador de energía, es conocida su aplicación en los dispositivos de máquinas herramientas, estampado, perforación, empaques y otras aplicaciones; propósitos que se consiguen empleando la energía de un fluido que se transmite con el movimiento por medio de la presión de un líquido (aceite 0 aire, a veces agua).

Asimismo, su aplicación es de importancia académica en los procesos de manufactura por deformación plástica, ensayo de materiales, prueba de instalaciones para conducción de fluidos y otros procesos automatizados.

\section{O B J E T I V O S}

Se consideran como objetivos:

- Diseñar un sistema hidráulico para la generación de energía.

- Lograr aplicaciones prácticas a los conocimientos teóricos.

- Fortalecer los conocimientos mediante pruebas experimentales en la aplicación y uso de energía.

- Integrar con el proyecto la disponibilidad de equipos y maquinarias de la Facultad.

H I P Ó T E S I S

a. ¿El mecanismo se moverá según lo deseamos que se mueva?. La respuesta se obtendrá mediante el análisis cinemático del sistema.

b. ¿Qué tamaño tendrá el pistón?. Dependerá de la magnitud de la fuerza que debe transmitir.

c. ¿Qué tan rápidamente se moverá el pistón?. La respuesta se propone en función del caudal del fluido del sistema.

d. ¿Qué tamaño y qué clase de bomba se debe utilizar?. Dependerá del caudal y la presión.

e. ¿Son necesarios equipos operacionales?. Esta interrogante incluye a: - Seguridad.

- Manual de operación y entrenamiento.

- Procedimientos de mantenimiento. 
CONSIDERACIONES DEL MECANISMO DE F U N C I O N A I E N TO

En el esquema mostrado en la Figura 1 se ilustra la forma física que deberá tener el sistema hidráulico, cuya característica es como sigue: una masa de fluido localizado en el punto 1 pasa por la bomba a través de la válvula entrando en el cilindro y produce el trabajo empujando el pistón. Como resultado del movimiento del pistón, el fluido en el compartimiento del cilindro y a baja presión [3] es empujado de nuevo al depósito (tanque). Los estados de flujo en 1 y 2 deberán ser relacionados mediante un modelo matemático de la energía fluida del sistema, lo cual marcan las pautas para el diseño.

En el sistema indicado es importante la capacidad de la bomba para mover el pistón contra una carga especificada. Esto es, en el contexto del diseño del sistema de energía fluida, significará encontrar la respuesta a lo siguiente:

a. Presión de sistema

b. Área del pistón

c. Diámetro y espesor del cilindro del pistón

d. Diámetro del cilindro vástago

e. Velocidad del pistón

f. Caudal de la bomba

g. Requisitos de energía para la bomba

Los parámetros a considerar son:

- Velocidad del fluido

- Presión

- Régimen del flujo.

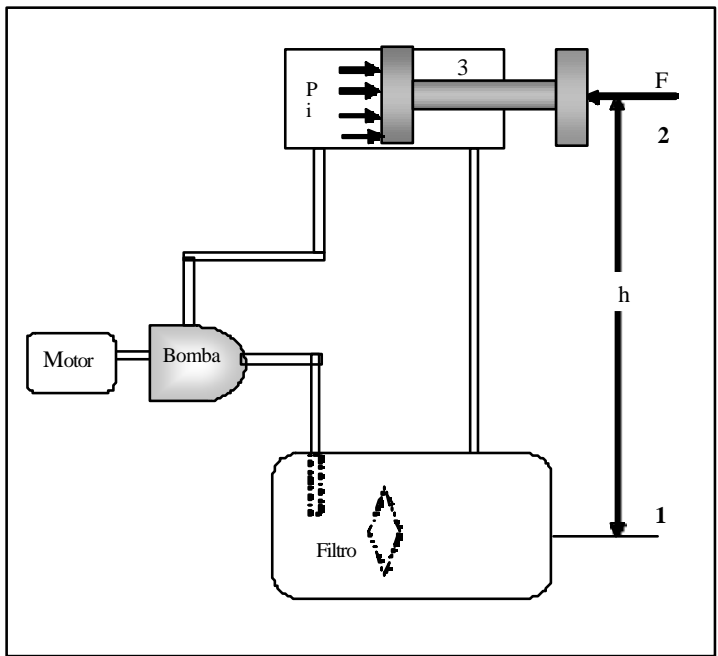

Figura 1. Esquema del sistema hidraúlico Fuente: Elaboración propia, 2005
Los cuales dependen principalmente de las características del fluido a emplear, que se describen a continuación:

Consideraciones respecto al fluido Un fluido usado en un sistema hidráulico debe cumplir muchas funciones críticas, tal como servir de medio para la transmisión de energía, actuar como lubricante, sellante, y medio de transferencia térmica. También debe maximizar la potencia y eficiencia minimizando el desgaste. El fluido con estas características es el aceite cuyas características físicas relevantes para su selección son:

a. Gravedad específica, siendo necesaria evaluar la gravedad específica de los fluidos y/o su importancia para su empleo.

b. Módulo a granel, esta propiedad depende del grado de compresibilidad del fluido, es el factor de importancia para la transferencia más eficiente de la energía; el efecto crítico en módulo a granel es la presencia de burbujas. El módulo a granel se evalúa mediante la relación siguiente:

$$
\beta=-\Delta p \frac{V}{\Delta V}
$$

El módulo a granel para el aceite nuevo es alrededor de $1500 \mathrm{MPa}$.

Las burbujas de aire en aceite tienen un efecto crítico en módulo a granel según la relación siguiente:

$$
\frac{1}{\beta_{\text {efec. }}}=\frac{1}{\beta_{\text {aceite }}}+\frac{V_{\text {aire }}}{V_{\text {aceite }}} \frac{1}{p}
$$

c. Viscosidad, propiedad que influye en la operación del sistema, consumo de energía, recalentamiento y desgaste de accesorios. La viscosidad del fluido debe ser lo suficientemente alta para asegurar una película fluida entre las superficies móviles, pero no tan grande como para crear excesiva fuerza friccional reduciendo la potencia de salida. En efecto la viscosidad, es la propiedad del fluido de oponerse a su flujo cuando se le aplica una fuerza.

La fuerza por unidad de área que hay que aplicar es proporcional al gradiente de velocidad. La constante de proporcionalidad se denomina viscosidad $\mu$.

$$
\frac{F}{A}=\mu \frac{d v}{d x}
$$


Energía en el sistema hidráulico Sea una partícula fluida en 1a (Figura 1) que pasa por la bomba a través de la válvula, entrando al cilindro y produce el trabajo empujando el pistón. Como resultado del movimiento del pistón, el aceite en el compartimiento de baja presión se empuja de nuevo al depósito. Los estados del flujo en 1 y 2 son relacionados por la ecuación siguiente:

$$
\begin{gathered}
Z_{1}+\frac{p_{1}}{\rho g}+\frac{V_{1}^{2}}{2 g}+h_{p}-h_{m}-h_{f}=Z_{2}+\frac{p_{2}}{\rho g}+\frac{V_{2}^{2}}{2 g} \\
h_{p}=\frac{W_{p}}{\rho g Q} \quad h_{m}=\frac{W_{m}}{\rho g Q} \quad h_{f}=f \frac{L}{d} \frac{V^{2}}{2 g} \\
f=\frac{64}{\operatorname{Re}} \quad \operatorname{Re}=\frac{V d}{v}
\end{gathered}
$$

Donde:

Re, número de Reynolds

$\mathbf{V}$, velocidad

d, es el diámetro del cilindro (tubo)

$\boldsymbol{\eta}$, viscosidad cinemática.

La altura de elevación y la velocidad se ignoran, lo que permite simplificar y tener la relación siguiente: $\mathrm{h}_{\mathrm{p}}=\mathrm{h}_{\mathrm{m}}+\mathrm{h}_{\mathrm{f}}$

Nota: La mayoría de los sistemas hidráulicos se diseñan para funcionar en el régimen de flujo laminar $(\operatorname{Re}<2000)$.

Régimen de flujo laminar

El flujo laminar es deseable debido a la fricción y ruidos bajos. El régimen del flujo se determina por un número sin dimensiones llamado número de Reynolds y este debe ser un número bajo para que el flujo sea sin turbulencia. El número de Reynolds se calcula mediante la siguiente relación:

$$
R_{e}=\frac{\rho D \mu}{v}
$$

El cual debe ser menor a 2000.

$$
\text { ACERCA DEL PISTÓN }
$$

Consideraciones técnicas

La construcción del piston hidráulico se fundamenta en el proceso iterativo del diseño, basado en los principios científicos y tecnológicos, uso de los modelos matemáticos, técnicas de expresión gráfica, ciencia de los materiales, mecánica de materiales, válidos para determinar y establecer los índices y parámetros tecnológicos, así como los econométricos para predecir los índices económicos y de explotación.

Los índices tecnológicos a considerar son la resistencia mecánica, fiabilidad, rigidez y estabilidad de la estructura y los elementos que componen el equipo, mediante los modelos matemáticos se determinan la resistencia mecánica en el estado de esfuerzo, las deformaciones y el efecto que causa el cambio térmico.

Los materiales deben tener las cualidades adecuadas que respondan satisfactoriamente a las condiciones expuestas, para ello se procede a la clasificación, selección y control de las propiedades y por consiguiente la calidad de los materiales, que son de cumplimiento obligatorio según normas técnicas.

Los índices económicos y de explotación, serán criterios en lo concerniente a los recursos de longevidad, largos periodos entre reparaciones, reducción de los gastos de explotacion.

Con la combinación de recursos tecnológicos y económicos, se logra la construcción del equipo con coeficiente de eficiencia apropiado.

Según las normas técnicas las cargas a considerar son:

$$
F=\Sigma P_{m}+\Sigma P_{v}+F_{w}+\Sigma P_{d i v}
$$

Los esfuerzos son:

\section{a. Estado de esfuerzo:}

$$
\begin{aligned}
& e_{x}=\frac{\sigma_{x}}{E}-\frac{v \sigma_{y}}{E}-\frac{v \sigma_{z}}{E} \\
& e_{y}=\frac{\sigma_{y}}{E}-\frac{v \sigma_{x}}{E}-\frac{v \sigma_{z}}{E} \\
& e_{z}=\frac{\sigma_{z}}{E}-\frac{v \sigma_{y}}{E}-\frac{v \sigma_{x}}{E}
\end{aligned}
$$

b. Por pandeo, para conocer el comportamiento del vástago por accion de la carga axial:

$$
\begin{gathered}
E I \frac{d^{2} y_{1}}{d x^{2}}=P\left(f-y_{1}\right) ; \quad o \leq x \leq \frac{l}{2} \\
E I \frac{d^{2} y_{2}}{d x^{2}}=P\left(f-y_{2}\right)-\frac{2 P f}{l}\left(x-\frac{l}{2}\right) ; \quad \frac{l}{2} \leq x \leq l
\end{gathered}
$$




\section{Velocidad del pistón}

La rapidez de deslizamiento del pistón depende de su tamaño y del porcentaje de aceite que fluya dentro del cilindro. Para establecer la relación porcentual entre el flujo y la velocidad, se considera el volumen que debe llenarse en el cilindro para efectuar un deslizamiento especificado, bajo las siguientesconsideraciones:

- La fuerza o torsión de un actuador es directamente proporcional a la presión e independiente del flujo.

- La velocidad o porcentaje de movimiento dependerá de la cantidad de flujo de fluido sin tomar en cuenta la presión.

consideraciones económicas

El rendimiento de la máquina en un periodo de tiempo, es la eficiencia del trabajo útil en un tiempo dado, su magnitud depende de la productividad y relacionado con el número de operaciones.

Los factores principales del rendimiento económico son la eficiencia, la longevidad, la fiabilidad, el costo de la mano de obra, el consumo de energía, el costo de las reparaciones y el costo de la fabricación de la máquina.

$$
E_{f}=N_{P}(v)
$$

\section{Gastos de explotación}

Referido para todo el periodo de trabajo: Amortización de la máquina, Am; Energía consumida, Ec; Materiales consumidos, Mc; Mano de obra, Mo; Mantenimiento, Man; Gastos accesorios y Gac; Gastos de reparación, Rep.

$G=A m+E c+M c+M o+M a n+G a c+\operatorname{Re} p$

\section{Rentabilidad de la máquina}

La rentabilidad Re se comprobará por la eficiencia Ef en un determinado periodo de tiempo y la suma de los gastos $\mathrm{G}$ en la explotación durante el mismo periodo.

$$
R_{e}=\frac{E_{f}}{G}
$$

\section{Renta - Efecto económico}

El efecto económico anual de la máquina (renta anual)es:

$$
Q=E f-G=E f\left(1-\frac{G}{E f}\right)=E f\left(1-\frac{1}{R_{e}}\right)
$$

Con el diseño se ha determinado y comprobado los parámetros siguientes:
Longitud total de tuberías: $7 \mathrm{~m}$

Potencia de la Bomba: $10 \mathrm{~kW}$

Caudal : $120 \mathrm{l} / \mathrm{min}$

En base a estos, se tiene:

Diámetro de tuberías: $25 \mathrm{~mm}$

Presión interna es: 5,0 MPa

Partes del pistón

En la Figura 2 se muestra el ensamble del pistón, donde se indican sus partes:
A, Brida de fijación
$B$, Cilindro
C, Disco de deslizamiento
D, Pistón
E, Anillo de tope
$F$, Aro de contención
G, Brida para sujeción de herramienta

Consideraciones del diseño La velocidad del pistón depende de los factores siguientes:

a. La rapidez de deslizamiento del pistón depende de su tamaño y el porcentaje de aceite que fluye dentro del cilindro.

b. La fuerza del pistón es directamente proporcional a la presión e independiente del flujo.

c. La velocidad de movimiento depende de la cantidad de flujo de fluido sin considerar la presión.

Por consiguiente para el dimensionado del pistón, se consideran:

- Estado de esfuerzo

- Por pandeo

- Propiedades del material

Por tanto el diámetro del pistón resulta igual a $120 \mathrm{~mm}$.

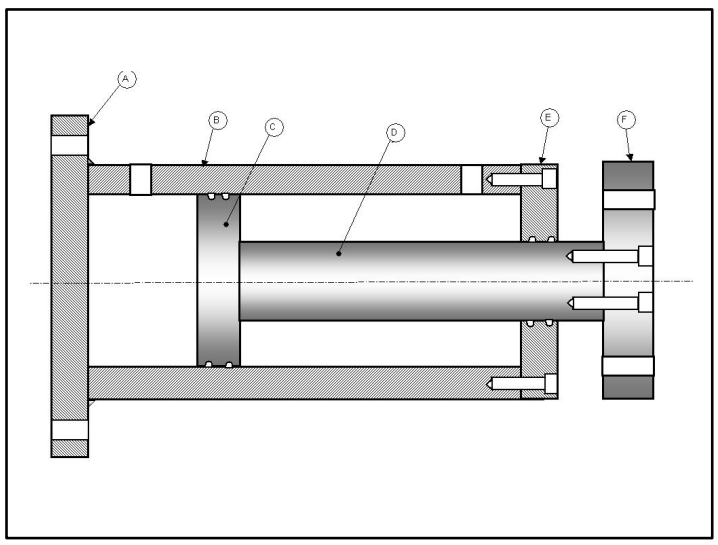

Figura 2. Esquema del pistón Fuente: Elaboración propia, 2005 


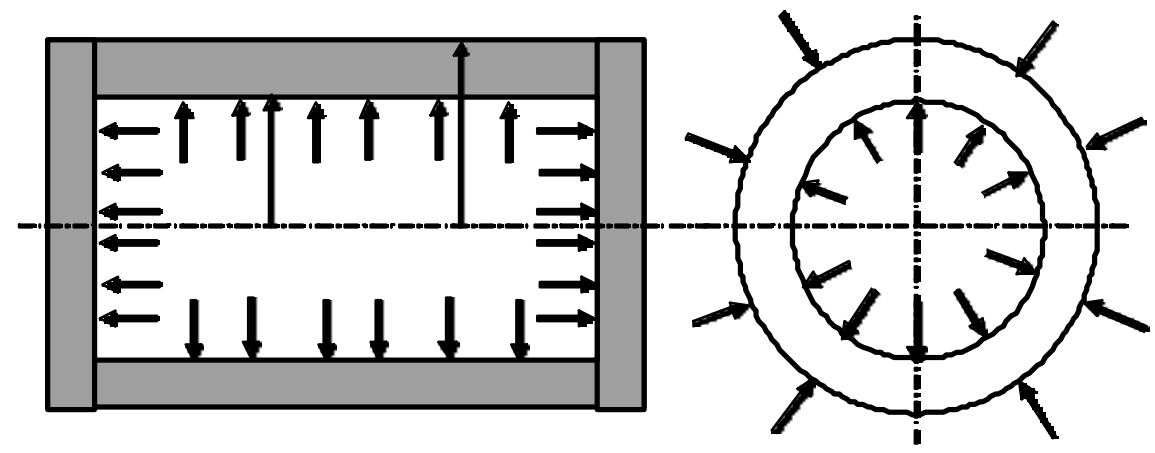

a)

b)

Figura 3. Presión del cilindro

Dimensionado del Cilindro

El dimensionado del cilindro y el vástago se considera según el esquema de la figura $3 a$ y b, dependiente de los parámetros siguientes:

a. Presión interna $P_{i}$

b. Presión externa $\mathrm{p}$ 。

c. Carga axial

d. Cambio térmico $\Delta \mathrm{T}$

Los esfuerzos y las deformaciones se deben a la presión ejercida por el fluido en la pared interna del cilindro según se muestra en la Figura 3, enseguida se muestran las relaciones para los esfuerzos y deformaciones.

RelacionesEsfuerzo-Deformación-Temperatura

$\varepsilon_{r r}=\frac{1}{E}\left[\sigma_{r r}-v\left(\sigma_{\theta \theta}+\sigma_{z z}\right)\right]+\alpha \Delta T$

$\varepsilon_{\theta \theta}=\frac{1}{E}\left[\sigma_{\theta \theta}-v\left(\sigma_{r r}+\sigma_{z z}\right)\right]+\alpha \Delta T$

$\varepsilon_{z z}=\frac{1}{E}\left[\sigma_{z z}-v\left(\sigma_{\theta \theta}+\sigma_{r r}\right)\right]+\alpha \Delta T=C t e$

Considerando que el cambio térmico no es significativo, por tanto la fuerza desarrollada sin considerar las pérdidas internas por el vástago del cilindro hidráulico en la carrera de empuje es función de la presión del fluido y del área de la sección interior del cuerpo se determina mediante la siguiente expresión:

$$
F=\frac{\pi d^{2}}{A} p_{i}
$$

La relación anterior, combinando con las expresiones del estado de esfuerzo, se tiene la expresión para el diámetro del cilindro:

$$
d_{i}=\sqrt{\frac{F \sigma}{4 p_{i}\left[\sigma-2 p_{i}\right]}}
$$

La relación anterior se optimiza para el diámetro del cilindro considerando la presión interior y las propiedades del material con el cual se construye el cilindro.

Conctus 500 nes

El valor mínimo del diámetro interior requerido por un cilindro hidráulico, para trabajar en condiciones de fuerza requerida y según las propiedades del material, se obtiene para un valor de la presión interior.

La sección transversal (espesor del cilindro) depende de la presión de trabajo.

La optimización del diseño de los cilindros hidráulicos en lo referente a peso y dimensiones depende de las propiedades del material con que se construirá. Asimismo, la rigidez del vástago depende de la propiedad del material y su sección.

B I B L I O G R A F Í A

1. Baumeister, T. (1986). Mark's Mechanical Engineers Handbook. 6ta Ed. McGraw Hill.

2. Timoshenko y MacCullough (1970). Elements of Strength of Materials. Ed. Van Nostrand. 